\title{
Síndrome de Bean y albinismo: reporte del primer caso en Ecuador
}

\author{
Oyuki Ortiz-Culcay ${ }^{1}$, Mónica Hernández-Lojano², Fabián Zurita-Alvarado² \\ Juan Roldan-Crespo ${ }^{3}$, Oswaldo Vasconez-Hatt ${ }^{4}$
}

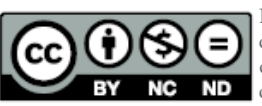

está bajo una cencia de Creative Commons de tipo Reconocimiento - No comercial - Sin obras derivaOPEN ACCESS das 4.0 International

1 Estudiante, Interna Rotativa de Medicina; Hospital Vozandes, Quito-Ecuador.

2 Médico, Residente Posgradista Medicina Familiar; Hospital Vozandes, Quito-Ecuador.

3 Médico, Tratante de Cirugía General; Hospital

Vozandes, Quito-Ecuador.

4 Médico, Tratante Medicina Interna; Hospital

Vozandes, Quito-Ecuador

ORCID ID:

Oyuki Dayanara Ortiz Culcay

https:/ / orcid.org/0000-0002-9512-6604

Mónica Patricia Hernández Lojano:

https:/ / orcid.org/0000-0002-4160-5212

Fabian Andrés Zurita Alvarado:

https:/ / orcid.org/0000-0002-7096-6594

Juan Roldán Crespo:

https:/ / orcid.org/0000-0001-5539-3452

Oswaldo Vasconez Hatt:

https:/ / orcid.org/0000-0001-5185-3546

Recibido: 8 - febrero - 2019

Aceptado: 14 - agosto - 2019

Publicado: 1 - noviembre - 2019

Correspondencia: Dr. Fabián Zurita A

E-mail: fzurita@hospitalvozandes.com

Contribución de autores: Los autores declaran haber contribuido de forma similar en la realización del manuscrito.

Financiamiento: Reporte financiado con fondos propios de los autores.

Conflictos de interés: Los autores declaran no poseer conflicto de interés.

\section{Resumen}

El síndrome de Blue Rubber Bleb Nevus (BRBNS) o su acrónimo Síndrome de Bean se caracteriza por malformaciones venosas (MV) distribuidas en múltiples zonas anatómicas, con predominio en piel y tracto gastrointestinal (Gl). La sintomatología se manifiesta comúnmente con episodios hemorrágicos y consecuente anemia crónica secundaria por déficit.

Se presenta el caso clínico de una paciente albina de sexo femenino de 68 años de edad, quien acudió por dolor abdominal agudo más rectorragia crónica, al realizar el examen físico llama la atención la presencia de malformaciones venosas (MV) caracterizadas por nódulos azulados, compresibles y heterogéneos, localizados en labio inferior, mentón, región perianal y extremidades, además de dolor a la palpación en hipocondrio derecho.

El análisis de laboratorio reveló valores de hemoglobina y hematocrito bajos y otros parámetros sugerentes de anemia por déficit de hierro, en el reporte de colonoscopia se lograron evidenciar múltiples malformaciones venosas $(\mathrm{MV})$, diseminadas con predominio rectal, el diagnóstico fue compatible con Síndrome de Bean por la asociación de MV en piel y tracto Gastrointestinal, además de anemia crónica secundaria.

El manejo fue clínico sintomático y quirúrgico paliativo, con una evolución favorable.

La asociación de albinismo y el síndrome de Bean no están reportadas en la literatura médica internacional, considerándose este el primer caso en Ecuador como una patología no muy frecuente, pero con complicaciones secundarias importantes. Se debería incluir este síndrome como una causa de malformaciones venosas inocuas, sangrado digestivo, anemia crónica y efectos oclusivos de vísceras huecas.

Palabras clave: Síndrome de Bean, Albinismo, Sangrado Gastrointestinal, Anemia, Síndrome de Hermansky Pudlak.

\section{Abstract}

\section{Bean Syndrome and Albinism: First Case Report in Ecuador}

Blue Rubber Bleb Nevus syndrome (BRBNS) or its acronym Bean syndrome is characterized by venous malformations (MV) distributed in multiple anatomical areas, predominantly in the skin and gastrointestinal (GI) tract. symptomatology is commonly manifested with hemorrhagic episodes and consequent chronic secondary anemia due to deficit.

We present the clinical case of a female albino patient of 68 years of age, who came for acute abdominal pain to this we add a chronic rectorrhagia, when performing the physical examination, the presence of venous malformations (MV) characterized by bluish, compressible and heterogeneous nodules, located in the lower lip, chin, perianal region and extremities, as well as pain on palpation in the right hypochondrium.

The laboratory analysis revealed low hemoglobin and hematocrit values and other parameters suggestive of iron deficiency anemia, in the colonoscopy report multiple venous malformations (MV) were disseminated, disseminated with rectal predominance, the diagnosis was compatible with Bean Syndrome by the association of MV in skin and Gastrointestinal tract and secondary chronic anemia.

The management was clinical symptomatic and palliative surgery, with a favorable evolution.

The association of albinism and Bean syndrome are not reported in the international medical literature, considering this the first case in Ecuador as a not very frequent pathology but with important secondary complications, this syndrome should be included as a cause of harmless venous malformations, digestive bleeding, chronic anemia and occlusive effects of hollow viscera.

Keywords: : Bean syndrome, Venous malformations, Albinism, Gastrointestinal Hemorrhage, Anemia, Hermansky Pudlak syndrome. 


\section{Introducción}

El síndrome de Blue Rubber Bleb Nevus (BRBNS), es muy raro y se caracteriza por malformaciones venosas (MV) distribuidas en múltiples regiones anatómicas, con predominio en piel, tejidos blandos y mucosas con especial afinidad por el tracto gastrointestinal (GI). ${ }^{(1,2)}$

Las lesiones cutáneas son generalmente pequeñas, circunscritas y rugosas a la palpación, su tamaño puede variar desde $1 \mathrm{~mm}$ hasta $10 \mathrm{~cm}$ de diámetro. ${ }^{(1,3,4)}$

Las lesiones gastrointestinales suelen estar localizadas por lo general en el intestino delgado (ID) y colon y su relevancia clínica es mayor que la de las lesiones de la piel, debido a las complicaciones que pueden desencadenar, tales como: anemia crónica por sangrado, intususcepción, vólvulo, hemorragia digestiva masiva, entre otras. ${ }^{(1,3)}$

El diagnóstico consiste en la asociación de lesiones en piel con exámenes endoscópicos que documenten la presencia de MV viscerales. $(1,3)$
Este síndrome puede ser heredado de forma autosómica dominante o presentarse de manera esporádica. $(1-3,5)$

Se trata de una entidad poco común, pues se han notificado 200 casos a nivel mundial 3 sin encontrar reportes en el país hasta la actualidad. Es llamativa, además su presentación en una paciente albina, condición heredable que, si bien predispone al desarrollo de otras patologías, resultaría atrayente descubrir relación existente entre estas dos enfermedades, asociación hasta el momento no confirmada. A continuación, presentamos un caso clínico-quirúrgico de Síndrome de Bean, considerado a su vez como el único caso documentado en Ecuador.
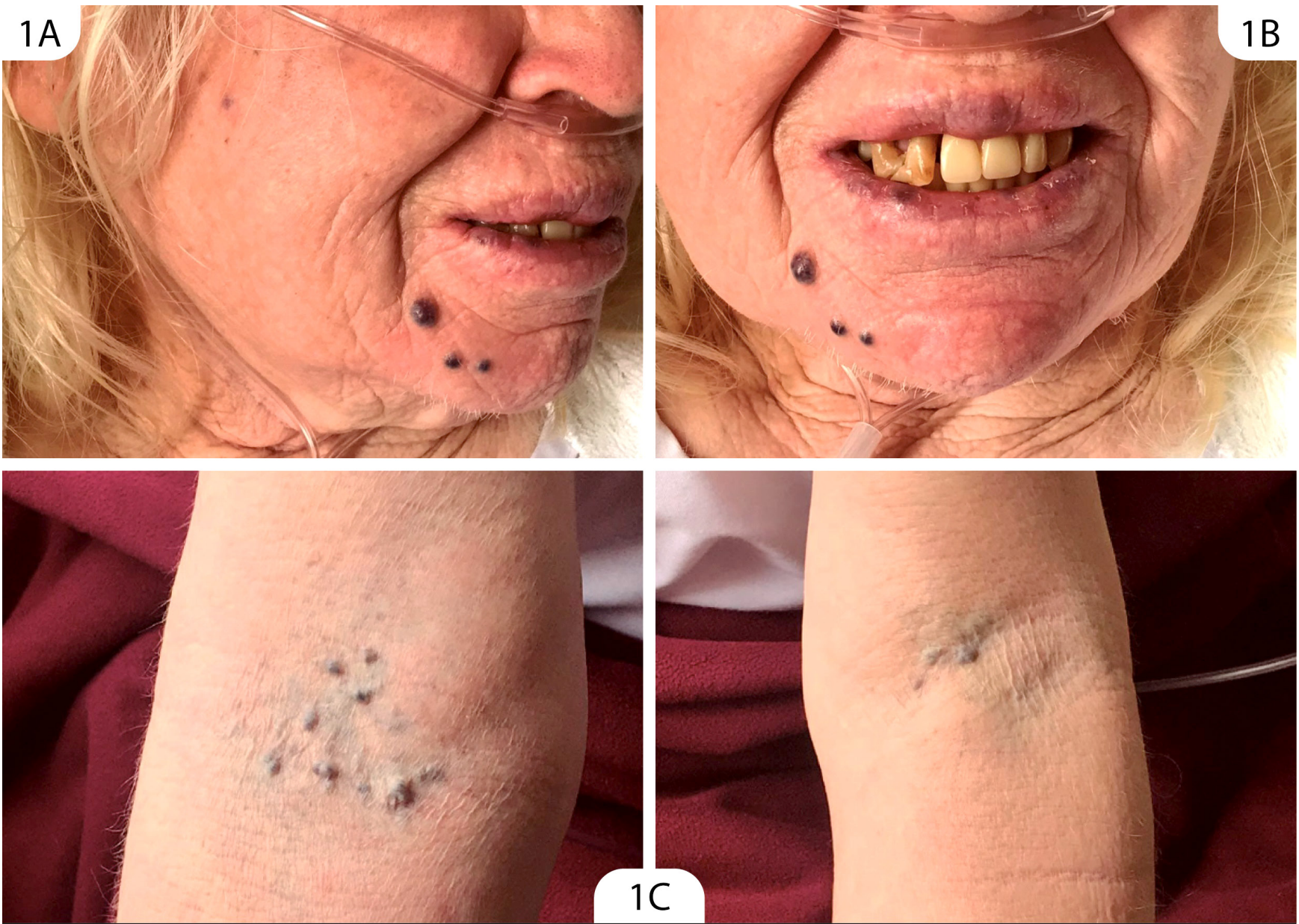

Figura 1A: Malformaciones mucocutáneas clásicas, características del síndrome de Blue Rubber Bleb - Nevus. Figura 1B: Lesiones vasculares venosas, violáceas, depresibles y no pulsátiles en labios y región mentioniana. Figura 1C: Múltiples nevus planos de bordes irregulares ubicados en cara interna de extremidades superiores. 


\section{Presentación de un caso}

Paciente de sexo Femenino de 68 años de edad, con antecedentes de albinismo óculo-cutáneo tipo II y trombosis colónica crónica, tratada con anticoagulación oral como único tratamiento durante cuatro meses. Acude a emergencia por presentar dolor abdominal súbito, postprandial, difuso, de moderada intensidad, nausea emetizante por 3 ocasiones; se acompañó de rectorragia moderada esporádica y gingivorragias ocasionales de 2 meses de evolución, que para la paciente resultaban ser normales. La paciente negó hematemesis, melenas, metrorragias o hemoptisis

Al examen físico se evidenció múltiples lesiones dérmicas nodulares, mucocutáneas, agrupadas, menores a $1 \mathrm{~cm}$, de contenido vascular, color violáceo, sin sangrado activo, localizadas en labio inferior, lado izquierdo de mentón, antebrazo distal bilateral y región perianal, su tiempo de evolución no se pudo determinar con precisión, sin recordar el tiempo exacto de aparición, mencionando simplemente su presencia desde hace algunos años atrás, sin causar molestia alguna que amerite su tratamiento inmediato (fotos 1A, 1B y 1C). En la exploración abdominal se halló dolor a la palpación profunda en hipocondrio derecho y sensación de masa palpable ipsilateral, el examen neurológico no reportó signos de focalidad ni lateralidad. El examen de laboratorio determinó hemoglobina: $7,6 \mathrm{~g} /$ dl, hematocrito: $30.3 \%$, volumen corpuscular medio (VCM): $80 \mathrm{fL}$, concentración media de hemoglobina ( $\mathrm{HbCM}): 28$ pg y plaquetas: $160.00 \mathrm{k} / \mathrm{ul}$; dímero D: $0.80 \mathrm{Ug} / \mathrm{ml}$, función hepática y renal normal, sangre oculta en heces negativo.

La radiografía abdominal reveló íleo intestinal marcado, sin niveles hidroaéreos, aire distal presente, materia fecal en marco colónico e imágenes múltiples calcificadas viscerales en la región pélvica (foto 2A Y 2B).

Se planteó como diagnóstico inicial trombosis mesentérica por su antecedente clínico, instaurando anticoagulación parenteral, analgesia y enema evacuante, 12 horas después el dolor se reagudiza decidiendo entonces valoración por cirugía general.

El examen físico complementario reveló la persistencia de dolor en hipocondrio derecho, signo de Murphy positivo y la presencia de una masa protruyente, no dolorosa de aproximadamente $2.5 \mathrm{~cm}$ de longitud en fosa iliaca izquierda, la tomografía abdominal mostró una vesícula biliar aumentada de tamaño, pared engrosada de $0.56 \mathrm{~cm}$ e imágenes hiperdensas múltiples en su interior sin efecto obstructivo, en colón descendente la presencia de múltiples imágenes polipoides intraluminales y estrechamiento de la luz en un segmento del sigma, calcificaciones granulomatosas en pared vesical y ampolla rectal, en fosa iliaca izquierda se apreció una hernia ventral encarcelada sin signos de isquemia (foto $3 \mathrm{~A}, 3 \mathrm{~B}, 3 \mathrm{C}$ y 3D), por los hallazgos y asociación se consideró como diagnóstico presuntivo, el síndrome Hermansky Pudlak.

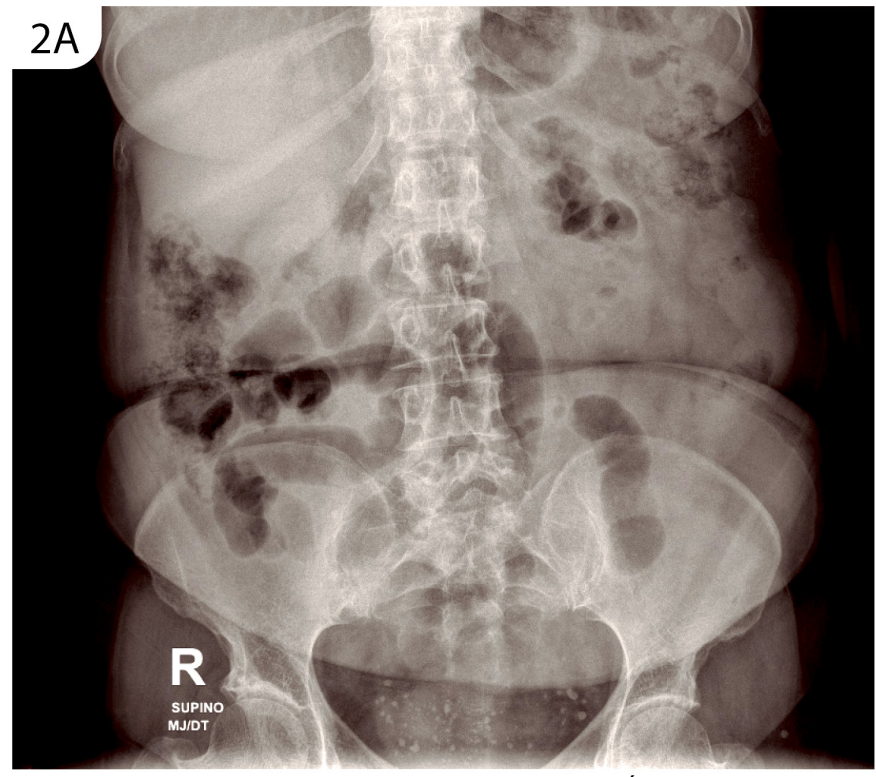

Figura 2 A: Radiografía de abdomen en supino. Íleo intestinal, ausencia de niveles hidroaéreos y presencia de aire en ampolla rectal.

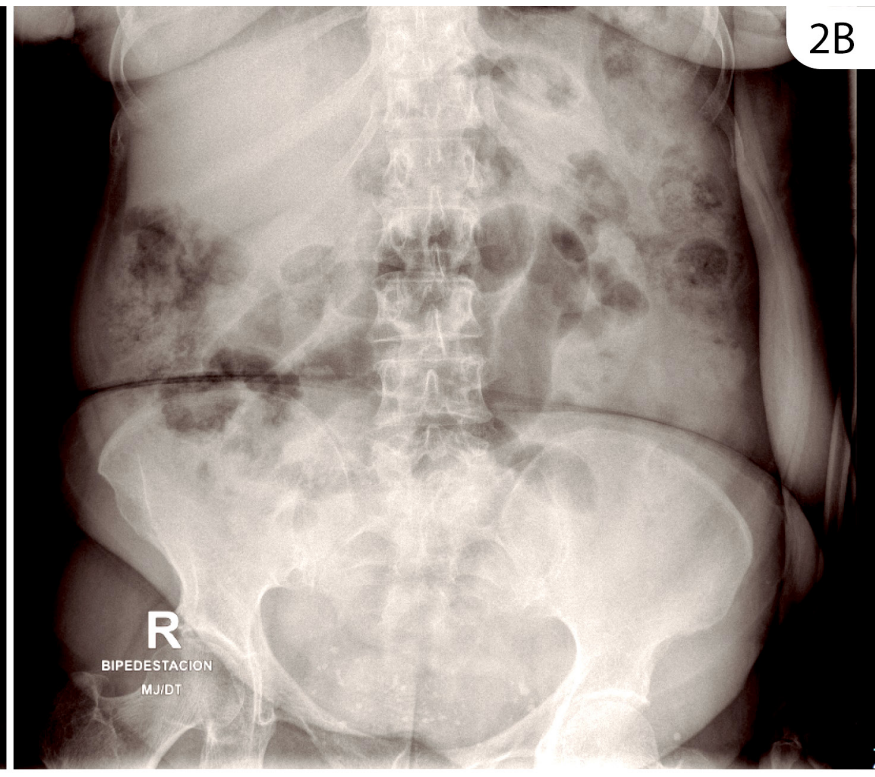

Figura 2 B: Radiografía de abdomen en bipedestación. Múltiples lesiones calcificadas en áreas viscerales y parietales intraabdominales. 

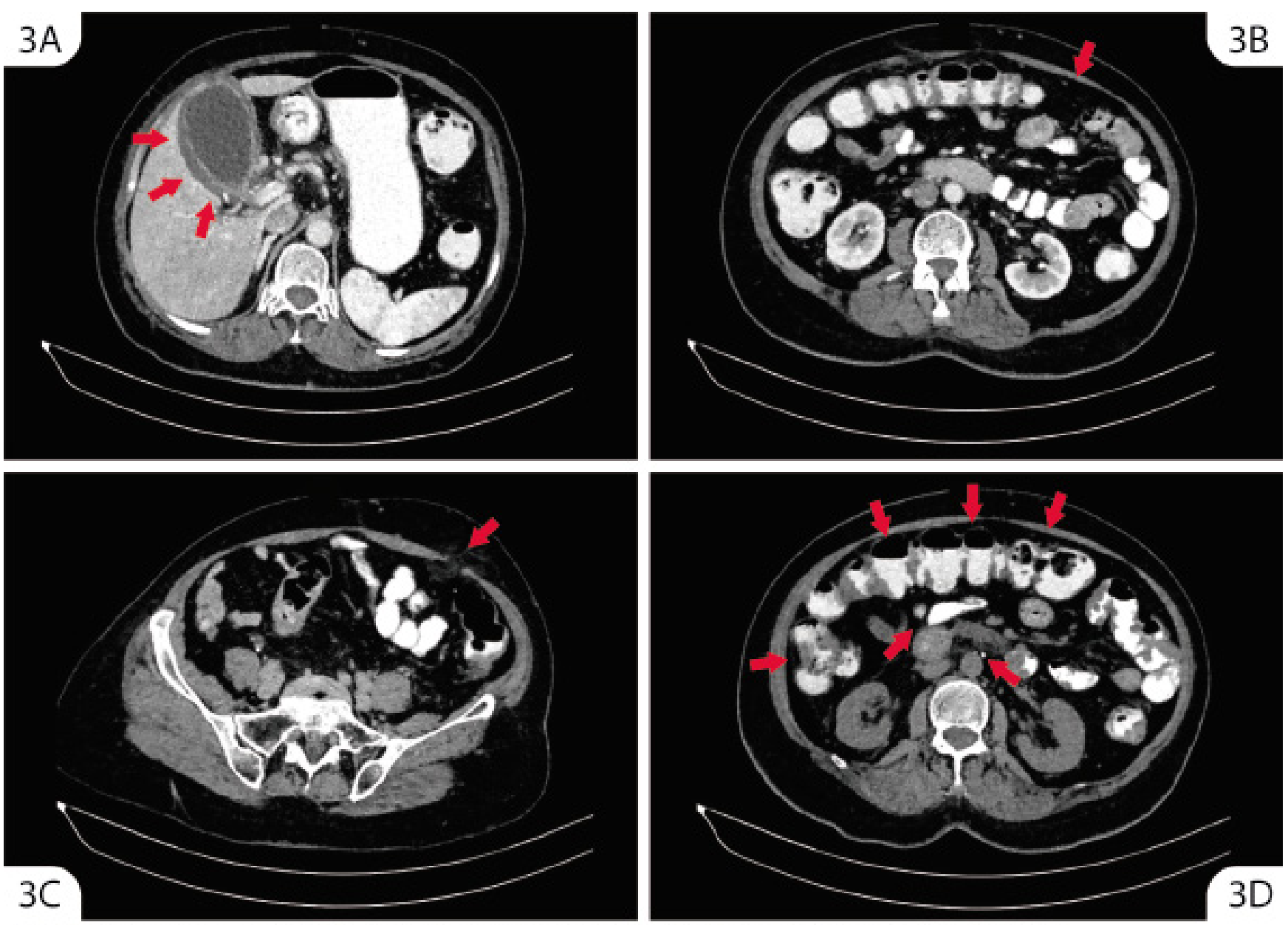

Figura 3A: Vesicula biliar hidrópica, pared engrosada, imágenes hiperdensas en su interior no liticas.

Figura 3B: Diverticulos esporádicos en colon descendente sin inflamación de grasa pericólica.

Figura 3C: Hernia abdominal de $1.1 \mathrm{~cm}$, contenido graso mesentérico, aparentemente encarcelada

Figura 3D: Calcificaciones granulomatosas en colon transverso, vejiga, sigma y ampolla rectal.

Al segundo día de hospitalización, la paciente presentó gingivorragia escasa continua y rectorragias de moderada cantidad por 6 ocasiones, signos vitales inestables con taquicardia que oscilaba entre 100 - 140 lpm., laboratorio determinó hemoglobina: 7 g/dl, hematocrito: $29 \%$ y plaquetas: $161.00 \mathrm{k} / \mathrm{ul}$., se consideró como una complicación de la patología de base, se suspendió la anticoagulación, indicando además transfusión sanguínea por dos ocasiones y requerimiento de examen endoscópico a las 48 horas.

Al cuarto día, con hemoglobina de control de $9 \mathrm{mg} / \mathrm{dl}$ se realizó la colonoscopia identificándose múltiples dilataciones violáceas de aspecto vascular, sangrado inactivo, diseminadas en todos los segmentos viscerales con predominio rectal más divertículos en sigma, hallazgos compatibles con síndrome Blue Rubber Bleb Nevus, (foto 4.1, 4.2, 4.3, 4.4, 4.5, 4.6, 4.7 y 4.8).

Una vez estabilizado el cuadro hemorrágico, se planificó cirugía paliativa con el objetivo de diferir complicaciones iniciales y a largo plazo: colecistectomía para erradicar el efecto oclusivo luminal ocasional de las MV y hernioplastia inguinal secundaria a debilitamiento de la pared abdominal, que aumentaría el riesgo de intususcepción o vólvulo intestinal. Los hallazgos importantes fueron presencia de nódulos vasculares venosos solitarios y/o agrupados localizados en parénquima hepático, vesícula biliar, colon y pared vesical, sin evidencia de signos isquémicos ni hemorrágicos. (foto 4)

Al séptimo día sin evidencia de sangrados y sin complicaciones ulteriores la paciente fue dada de alta, con hemodinamia estable, hierro oral y seguimiento por medicina interna y cirugía.

\section{Discusión}

El síndrome de BRBNS fue descrito en 1958 por William Bean, razón por la cual es citado como síndrome de Bean. ${ }^{(1-3)}$ 


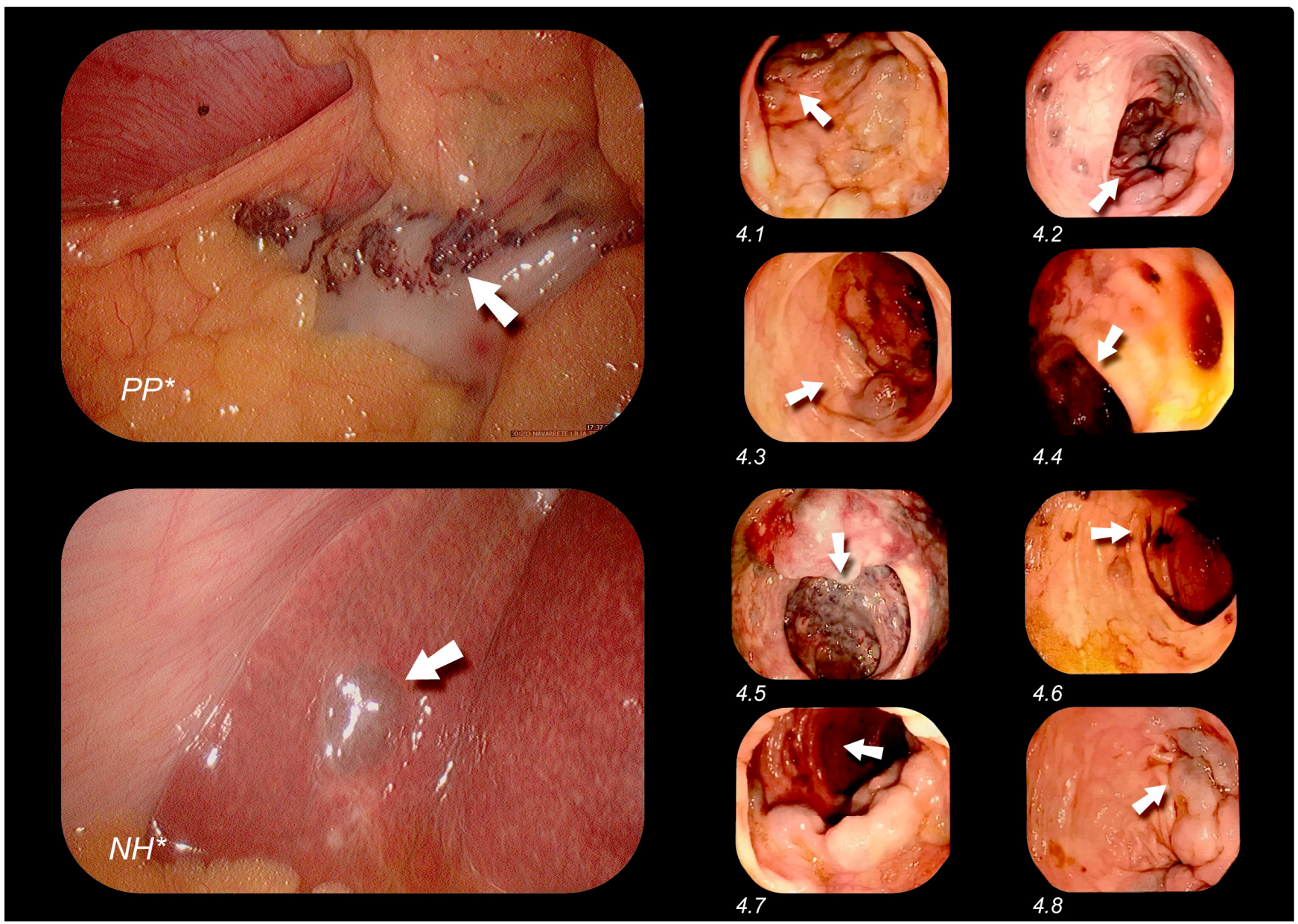

Figura 4: Dilataciones venosas viscerales. Visualización endoscópica que se confirma en procedimiento quirúrgico. 4.1 Ciego. 4.2 Colon ascendente. 4.3 - 4.4 Transverso. 4.5 - 4.6 Colon descendente. 4.7 Sigma. 4.8 Recto. $P P^{*}$ Peritoneo parietal: presencia de blue nevus. $\quad N H^{*}$ Nódulo hepático de $0.8 \mathrm{~cm}$ de diámetro en segmento 6 .

Las lesiones vasculares venosas sin potencial maligno determinado son MV simples, según la clasificación de la Sociedad Internacional para el Estudio de Anomalías Vasculares (ISVAS). ${ }^{(6)}$

Se han reportado 269 casos en el mundo y su etiopatogenia sigue siendo desconocida, se halló una asociación familiar vinculada a la mutación en el cromosoma 9p, sin embargo, la mayoría de casos pueden ser heredados de forma autosómica dominante o presentarse de forma esporádica siendo esta última la de mayor frecuencia, $(1,3,5)$ al igual que los casos reportados por Zahedi MJ, et al.8 y Chen W, et al. (7) no se evidenció ningún antecedente familiar ni asociación con otras condiciones congénitas como el albinismo. Por la negativa de la paciente, no fue posible realizar el estudio genético para determinar la mutación en el gen TEK del cromosoma $9 p$.

Jin $\mathrm{XL}$, et al. ${ }^{(3)}$ al igual que Choi KK, et al.5 coinciden con la bibliografía general, hombres y mujeres son afectados por igual, $(1,3)$ siendo la raza negra la menos involucrada, $(1-3,5)$ corroborado en este estudio y encontrándose de entre toda la bibliografía revisada un único caso en esta raza, reportado por Martínez CA, et al., (9) en nuestro caso la paciente es de raza mestiza y con herencia familiar de albinismo.

El caso de BRBNS que reportamos, presenta MV localizadas en piel de extremidades superiores, mentón, ano y labios, así como en múltiples vísceras, principalmente en el sistema $\mathrm{Gl}$. Jin $\mathrm{XL}$, et al. (3), realizaron un análisis de 200 casos, de estos 120 presentaron MV cutáneas y en 112 se apreciaron hemangiomas GI. Choi KK, et al.5, de un total de 39 pacientes, indica que la piel se vio afectada en 39 y el tracto $\mathrm{Gl}$ en 31, de la misma forma, como reportado por Chen $\mathrm{W}$, et al. ${ }^{(7)}$, uno de los sistemas más afectados es el tracto Gl, pues de un total de 5 pacientes, todos presentaron afección de este aparato, estas revisiones coinciden con la descripción del síndrome reportado en la bibliografía médica. ${ }^{(1,2)}$

Las MV pueden desarrollarse desde la boca hasta el ano y por lo general están localizadas en el intestino 
delgado (ID), teniendo mayor relevancia clínica por el riesgo de sangrado masivo que puede generar, (3) Texeira $M G$, et al. ${ }^{(10)}$, coinciden pues el ID fue el lugar donde mayor número de $\mathrm{MV}$ se encontraron, en su reporte de caso. A diferencia del nuestro, la localización mayoritaria fue en colon con predominio en recto, coincidiendo con el trabajo de Martínez CA, et al. (9) por esta razón consideramos que el diagnóstico inicial de trombosis colónica fue erróneo, probablemente por la observación de $M V$ que dio a la mucosa del colon un aspecto de trombosis.

Los nevus pueden desarrollarse a cualquier edad, $(1,3)$ en el estudio de Jin XL, et al. (3), se pudo identificar que el $30 \%$ de los casos tenían MV desde el nacimiento, el 9\% empezó en la infancia y adolescencia, y $4 \%$ en la edad adulta, sin embargo puede presentarse a cualquier edad, tal como lo menciona el caso de una paciente de 82 años, cuyas MV habían aparecido hace pocos años atrás, lo que coincide con lo descrito en nuestro caso, pues si bien se diagnostica a los 68 años, estas malformaciones también estuvieron presentes unos cuantos años antes, por su parte Gião Antunes AS, et al. (11), coinciden con nuestro caso en cuanto a la edad de diagnóstico (80 años), discrepando un tanto en cuanto al tiempo de aparición de las MV, pues en su caso se presentaron desde la infancia.

Las MV cutáneas son generalmente asintomáticas, pequeñas, circunscritas, depresibles, no pulsátiles, pudiendo aumentar con los años tanto en número como tamaño, su color varía de azul a púrpura, ${ }^{(13)}$ la descripción de las MV coinciden, en nuestro caso y no se identificó aumento de tamaño, mencionado por la paciente, a diferencia de los casos reportados por Kaur T, et al. ${ }^{(12)}$, y Zahedi MJ, et al. ${ }^{(8)}$ donde sí hubo crecimiento.

Las MV extra cutáneas pueden estar localizadas en el sistema nervioso central, ojos, nasofaringe, glándulas suprarrenales, riñón, corazón, pulmón, pleura, vejiga, útero, mesenterio, peritoneo, pene, tiroides, paratiroides, hígado, bazo y músculo esquelético. ${ }^{(1,3,4)}$ Recordemos que durante la intervención quirúrgica de la paciente, se evidencia la presencia de MV en hígado, vesícula, vejiga y peritoneo, similar a lo descrito por Chen W, et al. (7), donde uno de sus 5 casos reveló compromiso del hígado, mediastino, cavidad pélvica, cavidad abdominal y musculo ilíaco.

Si bien la anemia microcítica hipocrómica es la complicación más común de este síndrome, secundaria a pérdida (sangrado intestinal y deficiencia de hierro), en nuestro caso estamos ante una anemia normocítica normocrómica, justificada por la pérdida sanguínea gastrointestinal aguda, se decidió realizar niveles de hierro sérico en el contexto de una paciente con historia de sangrado Gl crónico.

La bibliografía menciona entre otras complicaciones la intususcepción, reportándose un caso por Deshpande GA, et al. (13) y otro por Menegozzo CAM, et al. (14), en una paciente embarazada, pero además se citan otras complicaciones: vólvulo, torsión y ruptura intestinal, ${ }^{(1,3)}$ en la revisión de Jin $\mathrm{XL}$, et al. ${ }^{(3)}$, de todos los casos analizados la mayoría de las lesiones $\mathrm{Gl}$ condujeron a hemorragia crónica y anemia por deficiencia de hierro, 7 casos desarrollaron intususcepción y un caso desarrolló gangrena, vólvulo e infarto, (2) considerando que la presentación de complicaciones no es un patrón, pues Fishmann SJ, et al. (4), en su estudio de 10 pacientes con síndrome de Bean, describe complicaciones en uno solo.

El sangrado gastrointestinal manifestado a través de gingivorragias, rectorragias y anemia secundaria sintomática, fueron las complicaciones iniciales en nuestro caso, sin embargo la alta probabilidad de oclusión de las vías biliares por la presencia de MV luminales y la encarcelación de un tejido intestinal de mala calidad con riesgo de sangrado no controlable, serían las complicaciones fatales para lo cual se tomó medidas paliativas radicales con éxito reportado hasta la redacción de este artículo.

Otras complicaciones menos frecuentes, dependen de la localización de las $\mathrm{MV}$, así se mencionan: infarto o hemorragia cerebral, compresión de la médula espinal y tos crónica. (12). Jin XL, et al. (3), también notifican otras complicaciones muy raras, tales como trastornos de la coagulación (4 casos), trombocitopenia (3 casos), coagulopatía intravascular diseminada (2 casos), sobre esta última, Menegozzo CAM, et al. (14), realizaron el reporte de una paciente embarazada, complicaciones que la paciente del presente caso no desarrolló.

El diagnóstico consiste en la asociación de MV en piel y aparato Gl, con o sin participación de otros órganos, episodios hemorrágicos agudos/ crónicos y anemia secundaria, ${ }^{(2,3)}$ se realizó el diagnóstico de BRBNS sobre la base de lo antes descrito, similar al trabajo realizado por Chen W, et al. ${ }^{(7)}$, en sus 5 casos, basado solamente en la clínica, 7 en contraste a nuestro caso Fishmann SJ, et al. (4), mencionan en su estudio, que de 10 pacientes solo uno de ellos no presentó MV en piel, 4 el examen endoscópico es el más práctico para reforzar el diagnóstico clínico, mediante la observación de las lesiones y valoración de riesgo de sangrado, (1-3) además se pueden realizar otros exámenes complementarios, de imagen sobre todo para evidenciar la presencia de MV en otros sitios anatómicos, sin embargo el uso de los mismos dependerá de la sintomatología que el paciente presente, ${ }^{(1,2)}$ en nuestro caso se decidió no complementar con otros exámenes, debido que la paciente no presentaba sintomatología adicional, a más de la descrita, que indique compromiso de algún otro órgano.

No existe tratamiento curativo descrito para esta patología hasta el momento, el manejo es conservador, con vigilancia de complicaciones adyacentes, las MV pueden ser tratadas precisando el riesgo de sangrado y localización a través de láser, escisión quirúrgica o escleroterapia, ${ }^{(1,3)}$ no se recomienda la resección 
de MV del aparato GI ya que es poco eficaz, debido a la cantidad de lesiones, riesgo hemorrágico y la probabilidad de recurrencia, este tipo de procedimiento está indicado en caso de sangrados masivos que comprometan la estabilidad hemodinámica del paciente, $(1,3,13)$ Choi KK, et al. ${ }^{(5)}$, Texeira ME, et al. ${ }^{(10)}$, y Fishman SJ, et al. ${ }^{(4)}$, han reportado excelentes resultados del tratamiento quirúrgico, en cuanto al control posterior del sangrado y anemia, nuestra paciente no requirió tratamiento quirúrgico y recibió tratamiento conservador.

En cuanto al tratamiento farmacológico los inmunosupresores como el sirolimus, han demostrado ser eficaces como anti angiogénicos al disminuir el tamaño de las $M V$ y reducir el sangrado gastrointestinal, haciéndose ya el reporte de un caso por Ogu UO, et al. ${ }^{(15)}$, donde se apreció buenos resultados en el tratamiento a largo plazo.

Si bien la literatura menciona que los principales diagnósticos diferenciales se los debería hacer con el síndrome de Osler-Weber-Rendu, Klippel-Trenaunay y Maffucci, enfermedades caracterizadas por diferentes formas de $M V$, ${ }^{(2,3,5,8,12)}$ los autores de este reporte de caso nos planteamos como principal entidad al síndrome de Hermansky-Pudlak tipo 2, por la asociación entre albinismo óculo-cutáneo y diátesis hemorrágica, esta última puede manifestarse con hematomas variables y hemorragias de distintos sitios anatómicos, pero además puede estar acompañado de fibrosis pulmonar, colitis granulomatosa o inmunodeficiencia. (16) Si bien la paciente presenta albinismo, gingivorragias y rectorragias, que nos sugería la posibilidad de diátesis hemorrágica, no existieron otros signos que indiquen trastornos de la coagulación ni comorbilidades características.

Finalmente, pocos son los casos reportados en la población latina, siendo el único a nivel nacional, es de importancia también considerar, que, si bien el albinismo favorece el desarrollo de otras patologías, aun no se ha logrado confirmar que este predisponga a la manifestación de síndrome de Bean, por lo que valdría la pena realizar investigaciones encaminadas a analizar esta asociación.

\section{Referencias}

1. Ballieux $F$, Boon LM, Vikkula $M$. Blue bleb rubber nevus syndrome. Handb Clin Neurol. rubber nevus syndrome. Handb Clin Neurol.
$2015 ; 132: 223-30$. doi: 10.1016/B978-0-444 2015;132:223-30. doi:
62702-5.00016-0. Review.

2. Carreño YA, Cárdenas MC, Cortés ER, Quizamán MA, Pardo MC, Toledo MB, et al. Síndrome de Bean: Artículo de revisión. Dermatología Cosmética, Médica y Quirúrgica. Octubre 2012 [citado 2019 Ene 12]; 10(4):290294. Available from: https://dcmq.com.mx/ images/ediciones/DCMQ_2011_V9-N2.pdf

3. Jin $X L$, Wang $Z H$, Xiao $X B$, Huang $L S$, Zhao $X Y$. Blue rubber bleb nevus syndrome: a case report and literature review. World J Gastroenterol. 2014 Dec 7;20(45):17254-9. doi: 10.3748/ wjg.v20.i45.17254. Review.

4. Fishman SJ, Smithers CJ, Folkman J, Lund DP Burrows PE, Mulliken JB, et al. Blue rubber bleb nevus syndrome: surgical eradication of gastrointestinal bleeding. Ann Surg. 2005 Mar;241 (3):523-8.

5. Choi KK, Kim JY, Kim MJ, Park H, Choi DW Choi $\mathrm{SH}$, et al. Radical resection of intestina blue rubber bleb nevus syndrome. J Korean Surg Soc. 2012 Nov;83(5):316-20. doi: 10.4174/ jkss.2012.83.5.316.

6. International Society for the Study of Vascular Anomalies. ISSVA classification for vascular
anomalies. 20th ISSVA Workshop. May 2018 [citado 2019 Ene 12]; Available from: https://
www.issva.org/UserFiles/file/ISSVA-Classification-2018.pd

7. Chen $W$, Chen $H$, Shan $G$, Yang $M$, Hu $\mathrm{Li} Q$, et al. Blue rubber bleb nevus syn-
drome: our experience and new endosdrome: our experience and new endos-
copic management. Medicine (Baltimore). 2017 Aug;96(33):e7792. doi: 10.1097/ MD.0000000000007792.

8. Zahedi MJ, Darvish Moghadam S, Seyed Mirzaei SM, Dehghani M, Shafiei Pour S, Rasti A. Blue Rubber Bleb Nevus Syndrome as a rare Cause of Iron Deficiency Anemia: a Case Re pause of Iron Deficiency Anemia: a Case Report and Review of Literat
Dig Dis. 2013 Oct; $5(4): 235-9$

9. Martinez CA, Rodrigues MR, Sato DT, Silveira Júnior PP, Gama RF, et al. CB, Pereira JA. Blue rubber bleb nevus syndrome as a cause of lower digestive bleeding. Case Rep Surg. 2014;2014:683684. doi: 10.1155/2014/683684

10. Teixeira MG, Perini MV, Marques CF, HabrGama A, Kiss D, Gama-Rodrigues JJ. Blue rubber bleb nevus syndrome: case report. Rev Hosp Clin Fac Med Sao Paulo. 2003 MarApr;58(2):109-12.

11. Gião Antunes AS, Peixe B, Guerreiro H. Blue Rubber Bleb Nevus Syndrome: A Delayed Diagnosis. GE Port J Gastroenterol. 2017 Mar;24(2):101-103. doi: 10.1159/000450876.

12. Kaur T, Singh $S$. Blue rubber bleb nevus syndrome: a case report. Indian J Dermatol.
2014 Jan;59(1):98-9. doi: 10.4103/00195154.123524

13. Deshpande GA, Samarasam I, George SV, Chandran S. Blue rubber bleb nevus syndrome: a rare cause of chronic gastrointestinal bleed in adults. Singapore Med J. 2014 Nov:55(1 1):e175-6.

14. Menegozzo CAM, Novo FDCF, Mori ND, Bernini $\mathrm{CO}$, Utiyama EM. Postoperative disseminated intravascular coagulation in a pregnant patient with Blue Rubber Bleb Nevus Syndrome presenting with acute intestingl obsdrome presenting with acute intestinal obstruction: Case report and literature review. Int J Surg Case Rep. 2017;:39:235-238. doi:
10.1016/j.ijscr.2017.08.026.

15. Ogu UO, Abusin G, Abu-Arja RF, Staber JM. Successful Management of Blue Rubber Bleb Nevus Syndrome (BRBNS) with Sirolimus. Case Rep Pediatr. 2018 Oct 8:2018:7654278. doi: $10.1155 / 2018 / 7654278$

16. Huizing $M$, Malicdan MCV, Gochuico BR Gahl WA. Hermansky-Pudlak Syndrome. 2000 Jul 24 [updated 2017 Oct 26]. In: Adam MP, Ardinger $\mathrm{HH}$, Pagon RA, Wallace SE, Bean LJH, Stephens K, Amemiya A, editors. GeneReviews $B$ [Internet]. Seattle (WA): University of Washington Seattle; from http://www.ncbi.nlm.nih.gov/books/
NBK1287/ 